Aims. The current pandemic and the restrictions on liberty that it has necessitated has had a huge impact on society as a whole. We were interested to learn how the constraints of sequential lockdowns and social distancing measures had affected inpatients in a mental health setting, many of whom were already contending with significant restrictions on their freedom.

Method. We conducted structured interviews with 24 service users across the Low Secure and Locked Rehabilitation Division at St Andrews Healthcare Northampton. We interviewed male and female inpatients with diverse diagnoses including emotionally unstable personality disorder, anorexia nervosa, schizophrenia and offending behaviours. All participants were detained under the Mental Health Act throughout the pandemic. Service users were asked the following questions:

How has the pandemic affected your mood?

How has it affected your relationship with your family?

How has it affected your treatment?

How has the pandemic affected your leave?

How has it affected how you use your free time?

Are there any other ways the pandemic has affected you?

We performed thematic analysis to identify ways the pandemic

has affected service users.

Result. Four major themes were identified:

1) Mental health

Participants reported a decline in mood.

2) Changing relationships

Service users reported that relationships with loved ones in the community had suffered from lack of contact and missing significant life events, however several participants felt that their relationships with peers had strengthened.

3) Delivery of care

Responses were split on the increased reliance on technology to replace face-to-face interaction between patients and team members, with some respondents reporting this as 'less intimidating', while others found this 'isolating'. Respondents reported reduced contact with MDT members and delays to recovery and step-down placements due to decreased leave.

4) Routine

Respondents reported an increase in free time throughout the pandemic. Some used this to develop hobbies whereas others reported becoming 'lazy' and expressed disappointment with the lack of exercise provision.

Conclusion. The pandemic has had significant emotional and psychological effects on society as a whole, but perhaps no group has been more affected than detained patients who have had their lives restricted to a massive degree. This group has been largely marginalised by government guidelines which often fail to consider individuals living in large group settings. By learning from the experiences of these service users we can adapt our practices to alleviate these issues in any future lockdowns and ensure our practices are the least restrictive possible.

\section{Social cognition and major depressive disorder: impact on psychosocial function and therapeutic opportunities}

\author{
Michael Weightman ${ }^{1 *}$ and Bernhard Baune ${ }^{2}$ \\ ${ }^{1}$ University of Adelaide and ${ }^{2}$ University of Melbourne \\ ${ }^{*}$ Corresponding author.
}

doi: 10.1192/bjo.2021.794
Aims. This poster aims to examine the impact of social cognitive deficits on psychosocial functioning in depressed patients, as well as summarise the utility of various evidence-based therapeutic interventions employed to target these deficits. The stated hypotheses were twofold: (1) that social cognitive impairment in major depressive disorder will correlate with poorer psychosocial functioning; and (2) that these deficits will respond to existing anti-depressant therapies.

Background. Social cognition is an important adaptive trait that incorporates the identification, perception and interpretation of socially relevant information from the external world. It is frequently affected in major depressive disorder such that depressed patien

Method. A review of the existing literature was performed in order to test the stated hypotheses. Pertinent sources were identified via the MEDLINE, EMBASE, PsycINFO, PubMed, Scopus and Google Scholar databases. A total of 107 studies met inclusion criteria for review.

Result. Impaired social cognitive performance in depressed patients correlated with poorer psychosocial functioning across the key domains of general cognitive functioning and quality of life. Many current anti-depressant therapies were found to have a normalising effect on the social cognitive abilities of depressed subjects, both at a neural and functional level. Anti-depressant medications, in particular citalopram and reboxetine, appeared to correct facial affect recognition deficits, while a psychotherapeutic approach demonstrated improvements in theory of mind and negative interpretive bias. Data relating to other common treatments, such as electroconvulsive therapy, are limited.

Conclusion. The impact and treatment of social cognitive deficits in major depressive disorder is an important emerging field. The social cognitive deficits evident in depressed patients are sometimes subtle, but afford a significant functional impact. Additionally, it appears these impairments are at least partially reversible using anti-depressants or psychotherapy.

\section{Aripiprazole as a risk factor for impulse control disorders: a systematic review}

Benjamin Williams ${ }^{1 \star}$, Benjamin Williams ${ }^{1}$, Kishen Neelam ${ }^{2}$ and Saumya Singh ${ }^{3}$

${ }^{1}$ Mental Health Liaison Team, Blackpool Victoria Hospital; ${ }^{2}$ Barnett House, Greater Manchester Mental Health NHS FT and ${ }^{3}$ Bolton

North CMHT, Bentley House, Greater Manchester Mental Health NHS FT

${ }^{\star}$ Corresponding author.

doi: 10.1192/bjo.2021.795

Aims. Aripiprazole is an anti-psychotic medication widely used for bipolar affective disorder and depression. It's primary mechanism of action is as a partial dopamine agonist. Aripiprazole's effect on dopamine signalling in the mesolimbic and mesocortical pathways may lead to impulse control disorders, as seen with other dopamine agonist medications. Aripiprazole is often chosen by prescribers because of its favourable side effect profile. There is a need to synthesise the available epidemiological literature on the potential association between aripiprazole use and impulse control disorders. This is needed to inform patients and prescribers of the best available evidence regarding this potential association. Our aim is to conduct a systematic review of the available non case-study evidence on the potential association between aripiprazole and impulse control disorders.

Method. Databases were searched using MEDLINE, PsychINFO, EMBASE, Cochrane Clinical Trials and Web of science. All 